\title{
Ehrlichiose canine en Tunisie. Enquête séroépidémiologique
}

\author{
A. Ghorbel ${ }^{1}$ B. Clerc $^{2}$ A. Djaiem ${ }^{3}$
}

GHORBEL (A.), CLERC (B.), DJAIEM (A.). Ehrlichiose canine cn Tunisie. Enquête séroépidémiologique. Revue Élev. Méd. vét. Pays trop., 1994,47 (3) : 271-275

La séroprévalence de l'ehrlichiose a été recherchée sur 1216 chiens de 8 zones administratives situées dans 5 régions à climat différent. Dans la zone de Tunis au sens large, où l'entretien des animaux cst satisfaisant, elle est de $\mathbf{4 9}$ p. 100 en moyenne alors qu'elle varie entre 75 et $85 \mathrm{p}$. 100 dans les autres régions de l'intérieur. Les auteurs soulignent l'importance de cette maladic et déerivent les conditions favorables à sa transmission tout en ajoutant que le pourcentage élevé de porteurs asymptomatiques prouve, s'il en est besoin, que Ehrlichia canis n'est qu'un germe de sortie en zone d'endémicité.

Mots clés : Chien - Ehrlichia canis - Prévalence - Epidémiologie Enquête - Sérologie - Transmission des maladies Endémie - Tunisie.

\section{INTRODUCTION}

L'ehrlichiose canine est une rickettsiose due à Ehrlichia canis, découverte en 1935 par DONATIEN et LESTOQUARD en Algérie (12). E. canis est transmise principalement par Rhipicephalus sanguineus, la tique brune du chien, mais d'autres tiques ont été incriminées dans la transmission de la maladie comme Otobius megnini (33). Cette rickettsie affecte les Canidés domestiques et sauvages $(23,25)$. L'infection humaine a été suspectée depuis 1987 aux États-Unis $(3,13,17,24,28,31)$ et en Tunisie (19). Cependant, en 1991, il a été démontré que l'ehrlichiose humaine n'est pas causée par $E$. canis mais par une nouvelle espèce, $E$. chaffeensis, tout au moins aux États-Unis $(2,11)$.

La maladie est de répartition mondiale ; elle a été diagnostiquée surtout dans les régions tropicales et semi-tropicales $(20,21)$ et dans l'hémisphère Nord dans les régions situées au-dessous du 45e parallèle (20). En 1982, KEEFE et al. (26) ont mené une enquête sérologique sur les chiens militaires américains à travers le monde et ont constaté une superposition des aires d'expansion de $R$. sanguineus et de l'ehrlichiose. Ils ont relevé notamment que le taux d'infection diminue en même temps que la température, passant de $13 p .100$ dans les zones tropicales et tempérées à $8 \mathrm{p} .100$ au-delà de $45^{\circ}$ de latitude nord.

1. Pathologie médicale des Équidés et des Carnivores, École nationale vétérinaire, 2020 Sidi Thabet, Tunisie.

2. Pathologie médicale des Équidés et des carnivores, École nationale vétérinaire, Lyon, France.

3. Docteur vétérinaire, Enfidha, Tunisie.

Reçu le 26.11.1992, accepté le 9.11.1994.
En Tunisie, $R$. sanguineus est présente sur tout le territoire occupant ainsi divers domaines bioclimatiques du sub-humide au semi-aride et saharien (8). Généralement, la maladie survient pendant la saison chaude de l'année (21) en se superposant aux fluctuations de $R$. sanguineus (26) ce qui correspond, en Tunisie, à la période allant des mois de mars à novembre (5). Mais la maladie peut être observée pendant la saison froide car la période qui sépare l'inoculation et l'apparition des premiers symptômes, lors d'une forme chronique, est relativement longue et ce, malgré l'absence de tiques au moment de l'examen (21). L'ehrlichiose peut sévir sous différentes formes:

- épizootique, surtout chez les chiens porteurs chroniques à la suite d'un stress collectif tel que la chaleur $(32,39)$;

- enzootique, survenant dans des chenils bien particuliers sur des chiens porteurs chroniques ou neufs $(7,8,9)$;

- sporadique, dans des zones infectées sur des animaux neufs ou affaiblis $(8,39)$ ou dans des régions indemnes, après un séjour du chien dans une zone infectée.

En Tunisie, la maladie a été mise en évidence $(5,7,15$, 18). Le but de ce travail est de connaître la séroprévalence de cette affection dans ce pays.

\section{MATÉRIEL}

\section{Animaux}

L'effectif total des animaux testés durant la période février-juin 1989 est de 1216 chiens répartis dans 8 zones administratives :

- 1ère zone : Le Grand Tunis comporte les gouvernorats de Tunis, Ariana et Ben Arous. Deux cent seize chiens y ont été testés après avoir été amenés à l'École nationale vétérinaire de Sidi Thabet, Service Maladies contagieuses, Zoonoses et Législation sanitaire, pour être vaccinés.

2e zone : (gouvernorat du Kef), 118 chiens.

3e zone : (gouvernorat de Béja), 138 chiens.

4e zone : (gouvernorat de Sousse), 249 chiens.

5 e zone : (gouvernorat de Sfax), 123 chiens. 
A. Ghorbel B. Clerc A. Djaiem

6e zone: (gouvernorat do Sidi Bouzid), 137 chiens.

7e zone : (gouvernorat de Kasserine), 128 chiens.

8e zone: (gouvernorat de Gabès), 107 chiens.

Pour ces 7 zones (carte 1), les prélèvements ont été faits chez les propriétaires à l'occasion d'une campagne de vaccination antirabique menée par le ministère de l'Agriculture. II n'a pas été tenu compte de la race, de l'âge et du sexe car il a déjà été démontré que ces facteurs ne jouent aucun rôle (36). En revanche, n'ont été pris en considération que les chiens cliniquement sains et âgés de plus de 3 mois (conditions de vaccination contre la rage).

II faut signaler que la population canine dans ces zones est estimée à 60000 chiens pour le Grand Tunis, 21000 pour le Kef, 40000 pour Béja, 30000 pour Sousse, 28000 pour Sfax, 60000 pour Sidi Bouzid, 60000 pour Kasserine et 48000 pour Gabès, soit 347000 sur un total général de 720000 chiens dans tout le pays (4).

\section{Sérums}

Les prises de sang ont été effectuées à la veine radiale, dans des tubes secs, par l'intermédiaire de seringues ou d'aiguilles "Veinoject". Le sang récupéré a été centrifugé et les sérums ont été stockés à $-20^{\circ} \mathrm{C}$ jusqu'à leur étude sérologique.

\section{Sérologie}

La sérologie a été effectuée à l'Ecole nationale vétérinaire de Lyon (France), au Service de Pathologie médicale des Equidés et des Carnivores pour les sérums de chiens de la zone de Sousse et à l'Ecole nationale vétérinaire de Sidi Thabet (Tunisie) pour les sérums de chiens des autres zones.

Cette sérologie a été faite selon la méthode de RISTIC et al (34). L'antigène est une suspension cellulaire (monocytes) préparée par culture cellulaire infectée par $E$. canis récoltée quand la moitié des cellules présentent des inclusions intracytoplasmiques au stade morula (fourni par E. VIDOR ${ }^{a}$ ). Cette suspension compte $2.10^{6}$ cellules $/ \mathrm{mm}^{3}$ et $10 \mu$ l sont déposés dans chaque puits d'une lame porte-objets Poly Labo ${ }^{b}$ comportant 36 puits répartis en trois rangées. Les lames sont séchées à la température ambiante, fixées à l'acétone à $-20^{\circ} \mathrm{C}$ pendant $20 \mathrm{~min}$, puis emballées dans du papier aluminium et stockées à $+4^{\circ} \mathrm{C}$ jusqu'à utilisation.

a. Laboratoire de parasitologie, Rhone-Mérieux, 254 rue Marcel Mérieux, 69007 Lyon, France.

b. Poly Labo Paul Block et Cie, 305 route de Colmar, 67160 Strasbourg, France.

c. PBS : Phosphate Buffered Saline-pH 7,2. Réf 7551-1 BioMérieux, 69292 Crapone, France.

d. Réf 7549-1 Bio-Mérieux, 69292 Crapone, France.

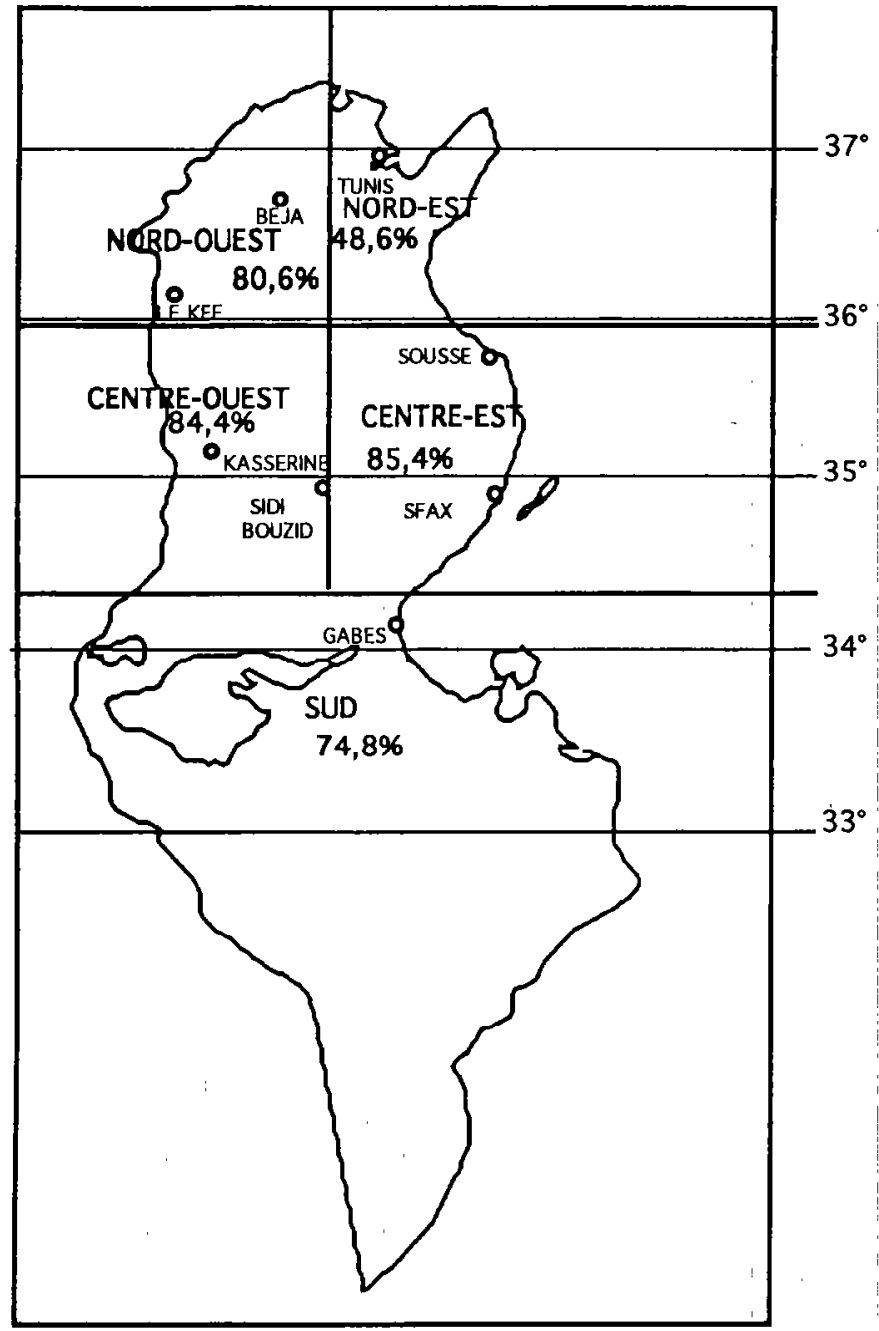

Carte 1 : Pourcentage des chiens séropositifs vis-à-vis d'E. canis dans les cinq régions d'étude.

Le test est spécifique pour une dilution du sérum au 1/10 (34). Cependant, WOODY et HOSKINS (40) soulèvent' l'hypothèse de l'existence d'une réaction antigénique croisée avec d'autres bactéries pour les sérums dont le titre est inférieur à 1/80. De même, DU PLESSIS et al. (14) montrent que $E$. canis croise très fortement avec Cowdria ruminantium mais cette rickettsie n'a pas été trouvée en Afrique du Nord et elle n'infecte pas le chien. DAWSON et EWING (11), pour leur part, prouvent que $E$. canis croise avec $E$. chaffeensis et cette dernière entraîne une maladie asymptomatique chez le chien. Dans un but de dépistage, on a donc considéré comme positifs tous les sérums présentant une fluorescence nette à une dilution supérieure ou égale à 1/80.

Les sérums sont dilués au 1/10 dans des tubes à hémolyse avec du PBS $^{c}(50 \mu l$ de sérum dans $450 \mu$ le PBS), puis au $1 / 80$ dans des plaques à microtitration à fond plat ${ }^{d}$. $10 \mu \mathrm{l}$ de chaque sérum dilué au 1/80 sont déposés dans chaque puits d'une lame porte-objets contenant 
préalablement de l'antigène fixé et sont incubés pendant $30 \mathrm{~min}$ à $37^{\circ} \mathrm{C}$. Deux lavages successifs de $5 \mathrm{~min}$ chacun, dans des bains de PBS et un rinçage pendant $1 \mathrm{~min}$ avec de l'eau distillée, sont ensuite effectués.

Après séchage à l'étuve à $37^{\circ} \mathrm{C}$ ou même au sèche-cheveux, $20 \mu \mathrm{l}$ d'un immunsérum de lapin anti-immunoglobuline $G$ de chien, conjugué à la fluorescéine (Cappel ${ }^{\ominus}$ ) dilué au 1/20 additionné de bleu d'Evans à 1 p. $100^{f}$ (une goutte dans $1 \mathrm{ml}$ ), sont déposés dans chaque puits et les lames sont mises de nouveau en incubation à $37^{\circ} \mathrm{C}$ pendant $30 \mathrm{~min}$.

Elles sont ensuite lavées 2 fois pendant 5 min dans du PBS et rincées pendant $1 \mathrm{~min}$ à l'eau distillée puis séchées. Les lames sont recouvertes d'une goutte de liquide glycériné Fluoprep ${ }^{9}$ (milleu de montage pour immunofluorescence) et d'une lamelle couvre-objets. Enfin, elles sont lues au microscope à fluorescence en lumière ultraviolette et au grossissement $400(10 \times 40)$. Un sérum contenant des anticorps dirigés contre $E$. canis donne une fluorescence nette des morulae. Un sérum négatif ne présente aucune fluorescence ou une fluorescence faible et diffuse affectant l'ensemble des structures cellulaires.

\section{RÉSULTATS}

La répartition des zones étudiées selon la région bioclimatique d'appartenance montre que le Grand Tunis se situe au nord-est, Béja et le Kef au nord-ouest, Sousse et Sfax au centre-est, Sidi Bouzid et Kasserine au centreouest et Gabès au sud (carte 1).

Si l'on compare le pourcentage des chiens séropositifs de ces 5 régions (tabl. I) avec un test $\chi^{2}$ avec 4 ddl, des différences significatives sont constatées. On remarque que le Centre-Est présente le taux maximum de chiens séropositifs (85,4 p. 100 suivi de près par le Centre-Ouest (avec 84,4 p. 100), puis le Nord-Ouest (avec 80,6 p. 100), le Sud (avec 74,8 p. 100), et enfin le Nord-Est (avec 48,6 p. 100). La comparaison de ces différentes régions deux à deux par le test de $\chi^{2}$ figure dans le tableau II.

\section{DISCUSSION}

Le recrutement des chiens n'a pas été fait de la même façon dans toutes les zones. En effet, les chiens qui ont été testés dans la région du Nord-Est proviennent de zones urbaine et rurale alors que dans les autres zones, les chiens vivent en milieu rural. II est à noter que les chiens vivant en milieu urbain sont mieux entretenus que ceux vivant en milieu rural. En outre, les chiens de la région du Nord-Est se sont déplacés jusqu'à l'Ecole vété-

e. Réf 12050082 Flobio Cappel, 15 rue Armand Silvestre, 92400 Courbevoie, France.

f. Réf. 7580-1 Bio-Mérieux, 69292, Crapone, France.

g. Réf. 7552-1 Bio-Mérieux, 69292, Crapone, France.
TABLEAU I Pourcentage des chiens séropositifs dans les 5 régions.

\begin{tabular}{|c|c|c|c|c|}
\hline Région & $\begin{array}{c}\text { Total } \\
\text { chiens }\end{array}$ & Positifs & Négatifs & $\begin{array}{c}\text { p. 100 } \\
\text { positivité }\end{array}$ \\
\hline Nord-Est & 216 & 105 & 111 & 48,6 \\
Grand Tunis & 256 & 206 & 50 & 80,6 \\
$\begin{array}{c}\text { Nord-Ouest Lentre-Est } \\
\text { Le kef, Béja }\end{array}$ & 372 & 320 & 52 & 85,4 \\
$\begin{array}{c}\text { Centre-Ouest Souse, Sfax } \\
\text { Sidi Bouzid, Kasserine }\end{array}$ & 265 & 224 & 41 & 84,4 \\
Sud Gabès & 107 & 80 & 27 & 74,8 \\
\hline
\end{tabular}

TABLEAU II Comparaison des pourcentages des 5 régions 2 à 2 par le test de $\chi^{2}$.

\begin{tabular}{|c|c|c|c|c|c|}
\hline & $\begin{array}{c}\text { Nord-Est } \\
48,60 \%\end{array}$ & $\begin{array}{c}\text { Nord-Ouest } \\
80,60 \%\end{array}$ & $\begin{array}{c}\text { Centre-Est } \\
85,40 \%\end{array}$ & $\begin{array}{c}\text { Centre-Ouest } \\
84,40 \%\end{array}$ & $\begin{array}{c}\text { Sud } \\
74,80 \%\end{array}$ \\
\hline $\begin{array}{c}\text { Nord-Est } \\
48,60 \%\end{array}$ & & $\begin{array}{c}52,8 \\
p<0,001\end{array}$ & $\begin{array}{c}95 \\
p<0,001\end{array}$ & $\begin{array}{c}70,9 \\
p<0,001\end{array}$ & $\begin{array}{c}20 \\
p<0,001\end{array}$ \\
\hline $\begin{array}{c}\text { Nord-0uest } \\
80,60 \%\end{array}$ & & & 3,45 & 1,33 & $\begin{array}{c}1,47 \\
\text { NS }\end{array}$ \\
\hline $\begin{array}{c}\text { Centre-Est } \\
85,40 \%\end{array}$ & & & & 0,27 & $\begin{array}{c}7,73 \\
\text { NS }\end{array}$ \\
\hline $\begin{array}{c}\text { Centre-Ouest } \\
84,40 \%\end{array}$ & & & & & $p<0,01$ \\
\hline Sud & & & & & 4,8 \\
$74,80 \%$ & & & & & \\
\hline
\end{tabular}

NS $=$ non significatif.

rinaire, ce qui prouve que, même s'ils proviennent d'une zone rurale, ils sont mieux suivis (du point de vue médical et hygiénique) par leurs propriétaires. Ainsi, le taux d'infection dans cette région est le plus faible, même s'il est de 48,6 p100, par rapport aux autres régions où la séroprévalence est très élevée, variant de 74,8 p. 100 dans le Sud à 85,4 p. 100 dans le Centre-Est.

L'influence de l'état d'entretien des chiens sur l'incidence de l'ehrlichiose peut s'exercer de deux manières :

- meilleure compétence immunitaire,

- plus faible risque d'infestation par les tiques.

L'existence de la maladie a été prouvée, en Tunisie, dans des chenils bien déterminés où le taux d'infection pouvait atteindre 100 p. 100 (18).

$E$. canis est transmise principalement par $R$. sanguineus qui existe sur tout le territoire tunisien et dont l'activité est 
continue durant toute l'année, avec un maximum de mars à novembre (6). Si la transmission du germe chez la tique ne se fait que de stade à stade, sans transmission transovarienne $(22,28,35)$, ce qui doit normalement diminuer l'ampleur épidémiologique de la maladie, la tique peut survivre jusqu'à 569 jours et transmettre la maladie pendant au moins $155 \mathrm{j}$ après un repas sanguin infectant (28).

D'après PYLE (32), le repas infectant ne serait possible que sur des chiens faisant une forme aiguë de la maladie et les porteurs sains ne constitueraient pas, semble-t-il, un réservoir du germe. C'est donc la longue période pendant laquelle la tique peut être infectante qui serait la seule explication épidémiologique des taux très élevés des chiens séropositifs dans les différentes zones étudiées. Par ailleurs, des études ont montré que $E$. canis persiste chez le chien plus de 29 mois après l'infection et probablement tout le long de la vie du chien (8), en l'absence de traitement $(1,8)$.

D'après cette étude, le Centre-Est et le Centre-Ouest paraissent les plus touchés $(85,4$ p. 100 et 84,4 p100 respectivement) par rapport au Nord-Ouest $(80,6 \mathrm{p} .100)$ et au Sud (74,8 p. 100), même si les conditions de l'enquête étaient identiques pour ces quatre régions. Ceci pourrait être attribué aux conditions climatiques. En effet, le NordOuest est une région montagneuse, le Sud bénéficie d'un climat saharien ; l'hiver est donc froid dans ces deux régions et la température peut descendre jusqu'à $0^{\circ} \mathrm{C}$ (condition non favorable à l'activité des tiques), alors que le Centre-Est et le Centre-Ouest sont des régions à climat doux pendant l'hiver et où les tiques restent actives (6). Or, l'enquête a été réalisée en partie pendant l'hiver dans les quatre régions.

Toutes les enquêtes épidémiologiques faites jusqu'à présent concernaient des chenils bien déterminés : des chenils militaires américains répartis dans différents pays du globe (26), ainsi que des chenils militaires en France $(9,10)$ et en Tunisie (18). La seule enquête effectuée sur toute une région a été menée par STEPHENSON et RISTIC (36) à Phoenix en Arizona. De plus, cette enquête concernait des chiens suspects de la maladie et des chiens apparemment sains, les résultats étaient respectivement de 14 et 6 p. 100 . A notre connaissance, aucune enquête séroépidémiologique de cette importance n'a été faite sur des chiens considérés comme porteurs sains et aucun résultat similaire n'a été publié.

Avec un tel taux d'infection, on peut se poser la question suivante : est-il possible d'attribuer l'ehrlichiose maladie à tout chien présentant un syndrome fièvre et une sérologie positive vis-à-vis de $E$. canis ?

Après une phase aiguë qui peut passer inaperçue $(20,21)$, l'infection peut rester à l'état latent, mais à la suite d'une dépression immunitaire quelconque telle qu'un stress $(30,36)$, une maladie intercurrente $(16,27$, 38) ou la vieillesse, la maladie réapparaît sous sa forme chronique et sa gravité dépend alors du statut immunitaire du chien essentiellement. S'agissant donc surtout d'une maladie de sortie, l'ehrlichiose doit être suspectée chez tout chien présentant un syndrome fièvre associé ou non à des troubles de l'hémostase, tout en recherchant également une maladie intercurrente éventuelle.

\section{CONCLUSION}

A partir de ce travail, on peut souligner l'importance probable de la séroprévalence de l'ehrlichiose en Tunisie puisque parmi les chiens testés, environ 3 sur 4 ont présenté un titre en anticorps supérieur ou égal à $1 / 80$, soit séropositifs. L'ehrlichiose canine est donc une maladie très répandue en Tunisie. Le pourcentage important des porteurs asymptomatiques permet de prouver une fois encore que $E$. canis n'est qu'un germe de sortie dans les zones endémiques. Cependant, sur des animaux de race introduite, la maladie peut s'exprimer cliniquement et elle est parfois mortelle.

\section{REMERCIEMENTS}

Nous remercions le Docteur E. VIDOR du Laboratoire de Parasitologie de l'Institut Mérieux qui nous a délivré la quantité d'antigène nécessaire à la réalisation de ce travail.

\section{BIBLIOGRAPHIE}

1. ACKAH (P.A.), MUSCAT (G.). Amélioration de l'efficacité de l'oxytétracycline dans le traitement des maladies infectieuses. Bull. mens. Soc. vét. prat. Fr., 1987, 71 : 49-58.

2. ANDERSON (B.E.), DAWSON (J.E.), IONFS (D.C.), WII SON (K.H.) E. chaffeensis a new species associated with human ehrlichiosis. J. Clin. Microbiol., 1991, 29: 2338-2342.

3. BARTON (L.L.), FOY (T.M.). Ehrlichia canis infection in a child. Pediatrics, 1989, 4: 580-582.

4. Bilan des trois premières années d'exécution du Programme National de Lutte Contre la Rage en Tunisie. Tunis, Ministère de l'Agriculture, Direction de la production animale, 1982. p. 83-84.

5, BOBIN (A.), CHABASSOL (C.), DE BRUX (J.). Syndrome hémorragique thrombotique et thrombopénique du chien en Tunisie. Revue Path. gén., 1962, 62: 317-334

6. BOUATTOUR (A.). Contribution à la connaissance des facteurs de distribution des tiques en Tunisie (Acariens , Ixodoidea). Thèse Doct. vét., Sidi Thabet, Tunisie, 1982.

7. BROUQUI (P.), DAVOUST (B.), HADDAD (S.), VIDOR (E.), RAOULT (D.). Serological evaluation of $E$. canis infections in military dogs in Africa and Reunion Island. Vet. Microbiol., 1991, 26: 103-105.

8. BUHLES (W.C.), HUXSOLL (D.L.), RISTIC (M.). Tropical canine pancytopenia: Clinical, hematologic and serologic response of dogs to Ehrli chia canis infection, tetracycline therapy and challenge inoculation. $J$. infect. Dis., 1974, 130: 357-367.

9. DAVOUST (B.), MACKOWIAK (M.), MOREAU (Y.). Ehrlichiose canine : enquête épidémiologique. Recl Méd. vét., 1986, 162: 471-475.

10. DAVOUST (B.), PRAZY (D.). Ehrlichiose canine : surveillance épidémiologique dans les chenils militaires du Sud-Est. Recl Méd. vét., 1989 165: $373-377$. 
11 DAWSON (J.E.), EWING (S.A.). Susceptibility of dogs to infection with E. chaffeensis, causative agent of human ehrlichiosis. Am. J. vet. Res., 1992, 53: 1322-1327.

12. DONATIEN (A.), LESTOQUARD (F.). Existence en Algéric d'une rickettsiose du chien. Bull. Soc. Path. exot., 1935, 6 : 418-419.

13. DORAN (T.I.), PARMLEY (R.T.), LOGAS (P.C.), CHAMBLIN (S.) Infection with Ehrlichia canis in a child. J. Pediat., 1989, 114: 809-812.

14. DU PLESSIS (J.L.), FOURNIE (N.), NEL (P.W.), EVEZARD (D.N.). Concurrent babesiosis and ehrlichiosis in the dog: blood smear examination supplemented by the indirect fluorescent antibody test, using Cowdria ruminantium as antigen. Onderstepoort J. vet. Res., 1990, 57: 151-155.

15. DURAN (M.). Notes sur l'hépato-néphrite hémorragipare du chien en Tunisie. Arch. Inst. Pasteur, Tunis, 1961, 38 : 33-50.

16. EWING (S.A.), BUCKNER (R.G.). Manifestations of babesiosis, ehrlichiosis and combined infection in the dog. Am. J. vet. Res., 1965, 26: 815-828.

17. FISCHBEIN (D.B.), SAWER (L.A.), HOLLAND (C.J.) et al. Unexplained febrile illnesses after exposure to ticks. J. Am. Med. Ass., 1987, 257: $3100-3104$.

18. GHORBEL (A.). L'Ehrlichiose canine. Enquête sérologique. Maghreb Vet. $1989,4: 5-8$.

19. GHORBEL (A.), KENNOU (M.F.), BEN HAMED (S.), BEN JEMAA (M.), VIDOR (E.). L'ehrlichiose humaine en Tunisie : Etude préliminaire. Méd. Mal. Infect., 1991, 21 : 725-731.

20. GREENE (C.E.). Rocky mountain spotted fever and ehrlichiosis. In: Current veterinary therapy small animal practice. 9e edn. London, W.B. Saunders Company, 1986. p. 1080-1084

21. GREENE (C.E.), HARVEY (J.W.). Canine elılichiosis. In: GREENE (C.E.). Clinical microbiology and infections diseases of the dog and cat. London, W.B. Saunders Company, 1984. p. 545-557

22. GROVES (M.G.), DENNIS (G.L.), AMYX (H.L.), HUXSOLL (D.L.). Transmission of Ehrlichia canis to dogs by ticks (Rhipicephalus sanguineus). Am. J. vet. Res., 1975, 36: 937-940.

23. HARVEY (J.W.), SIMPSON (C.F.), GASKIN (M.J.), SAMWEK (J.H.). Ehrlichiosis in wolves, dogs and wolf-dog crosses. J. Am. vet. Med. Ass., 1979, 175: 901-905.

24. Human ehrlichiosis in the United States. M.M.W.R., 1988, 37: 270, 275-277.

25. HUXSOLL (D.). Canine ehrlichiosis (tropical canine pancytopenia). A review. Vet. Parasitol., 1976, 2: 49-60.

26. KEEFE (T.J.), HOLLAND (C.J.), SALYER (P.E.), RISTIC (M.). Distribution of Ehrlichia canis among military working dogs in the world and selected civilian dogs in the United States. J. Am. vet. Med. Ass., 1982. 181: $236-238$

GHORBEL (A.), CLERC (B.), DJAIEM (A.). A seroepidemiological survey of canine ehrlichiosis in Tunisia. Revue Élev. Méd. vét. Pavs trop., 1994, 47 (3): 271-275

The seroprevalence of ehrlichiosis was investigated in 1,216 dogs from eight administrative areas in five regions with different climates. In and around Tunis, where the animals receive satisfactory care, the average prevalence is $49 \%$, but it ranges from 75 to $85 \%$ in the other regions of the interior. The authors underline the importance of this disease and describe the conditions which favour its transmission. They also note that the high percentage of asymptomatic carriers provides further proof that Ehrlichia canis is an opportunist pathogen in endemic areas.

Key words : Dog - Ehrlichia canis - Prevalence - Epidemiology - Survey Serology - Disease transmission - Endemics - Tunisia.
27. KROOSHOF (Y), HELLEBREKERS (L'J), FILDMAN (B.F.). Two cases of combined babesiosis and ehrlichiosis in dogs. Canine Practice, 1984, 11: 12-16

28. LEWIS (G.E.), RISTIC (M.), SMITH (R.D.), LINCOLN (T.), STEPHENSON (E.H.). The brown dog tick Rhipicephalus sanguineus and the dog as experimental hosts of Ehrlichia canis. Am. J. vet. Res., 1977, 38: 1953-1955.

29. MAEDA (K.), MARKOWITZ (N.), HAWLEY (R.C.), RISTIC (M.), COX (D), Mc DADE (JE.). Human infection with Ehrlichia canis, a leucocytic rickettsia. $N$. Engl. J. Med., 1987, 316: 853-856.

30. MOREL (P.C.), VASSILIADES (G.). Les Rhipicephalus du groupe sanguineus: espèces africaines (Acariens : Ixodoidea). Revue Élev. Méd. vét. Pays trop., 1962, 15 : 343-374.

31. PEARCE (C.J.), CONRAD (M.E.), FISCHBEIN (D.B.), DAWSON (J.E.). Ehrlichiosis: A cause of bone marrow hypoplasia in humans. Am. $J$. Hematol., 1988, 28: 53-55.

32. PYLE (R.L.). Canine ehrlichiosis. J. Am. vet. Med. Ass. 1980, 177: 1197-1199.

33. RIKIHISA (Y.). The tribe Ehrlichieae and ehrlichial diseases. Clin. Microbiol. Rev., 1991, 4: 286-308.

34. RISTIC (M.), HUXSOLL (D.L.), WEISIGER (R.M.). Serological diagnosis of tropical canine Pancytopenia by indirect immunofluorescence. Infect. Immun., 1972, 6: 226-231

35. SELLS (D.M.), HILDEBRANDT (P.K.), LEWIS (G.E.), NYINDO (M.B.A.), RISTIC (M.). Ultrastructural observations of Elrtichia equi organisms in equine granulocytes. Infect. Immun., 1976, 13: 273-280.

36. STEPHENSON (E.H.), RISTIC (M.). Retrospective study of an Ehrlichia canis epizootic around Phoenix, Arizona. J. Am. vet. Med. Ass., 1978, 172: $63-65$.

37. TAYLOR (J.P.), BETZ (T.G.), FISHBEIN (D.B.), ROBERTS (M.A), DAWSON (J.), RISTIC (M.). Serological evidence of possible human infection with Ehrlichia in Texas. J. infect. Dis., 1988, 158: 217-220.

38. UILENBERG (G.). FRANSSEN (F.F.J.), PERIE (N.M.), SPANJER (A.A.M.). Three groups of Babesia canis distinguished and a proposal for nomenclature. Vet. Q., 1989. 11: 33-40.

39. WALKER (J.S.). RUNDQUIST (J.D.), TAYLOR (R.), WILSON (B.L.) et al. Clinical and clinicopathologic findings in tropical canine pancytopenia. J. Am. vet. Med. Ass. 1970, 157: 43-55.

40. WOODY (B.J.), HOSKINS (J.D.). Ehrlichial diseases of dogs, Vet. Clin. North Am. Small Anim. Pract., 1991, 21: 75-98.

GHORBEL (A.), CLERC (B.), DJAIEM (A.). Erliquiosis canina en Túnez. Encuesta sero-epidemiológica. Revue Élev. Méd. vét. Pays trop., 1994, 47 (3) : $271-275$

Se buscó la seroprevalencia de la erliquiosis en 1216 perros, en ocho zonas administrativas situadas en cinco regiones de climas diferentes. En la zona de Túnez en general, en donde el mantenimiento de los animales es satisfactorio, es de 49 p. 100 en promedio, pero varía de 75 a 85 p. 100 en las otras regiones del interior. Los autores subrayan la importancia de esta enfermedad y describen las condiciones favorables para su transmisión, agregando a esto que el porcentaje elevado de portadores asintomáticos demuestra, si es del caso, que Ehrlichia canis es solamente un germen de salida en zonas endémicas.

Palabras clave : Perro - Ehrlichia canis - Prevalencia - Epidemiología Encuesta - Serología - Transmisión de enfermedades - Endemia - Túnez. 